International Journal of Pure and Applied Mathematics

Volume 93 No. 1 2014, 135-145

ISSN: 1311-8080 (printed version); ISSN: 1314-3395 (on-line version)

url: http://www.ijpam.eu

doi: http://dx.doi.org/10.12732/ijpam.v93i1.11

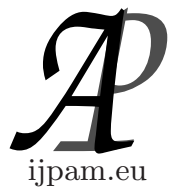

\title{
THREE-DIMENSIONAL SIMULATION THE RUNUP OF NONLINEAR SURFACE GRAVITY WAVES ON SHALLOW COAST
}

\author{
Iftikhar B. Abbasov \\ Taganrog Technological Institute \\ Southern Federal University \\ Nekrasovskyi 44, 347928, Taganrog, RUSSIAN FEDERATON
}

\begin{abstract}
The paper is devoted to three-dimensional numerical modeling of a runup of nonlinear surface gravity waves based on Navier-Stokes equations. The three-dimensional problem is stated, boundary and initial conditions are described. Using split method for physical processes, there has been constructed the discrete model with consideration of cell filling coefficient. The peculiarities of the modeled three-dimensional tank structure are described. There are presented three-dimensional models of stepwise runup of the surface gravity wave upon a sloping shore.
\end{abstract}

AMS Subject Classification: 76D33

Key Words: 3D numerical modelling of runup, Navier-Stokes equations, splitting method, shallow water tank, stepwise run-up of surface waves

\section{Introduction}

Within the frames of shallow water tanks, the shore formations are affected by constant wave action. The studies of shore lines change processes are vital for prediction and developing of the shallow water ecosystem. In describing wave phenomena, the various modifications of shallow water equations are often used. However, Navier-Stokes equations are most adequate in describing wave

Received: January 18, 2014

(c) 2014 Academic Publications, Ltd. url: www.acadpubl.eu 
processes. Those equations take into account both nonlinear processes and turbulent ones in viscous incompressible fluid.

Let us consider works on three-dimensional simulation of the surface waves' run-up onto the shore formations. The papers Watanabe \& Saeki, 1999, Lubin et all, 2006, are devoted to the three-dimensional simulation of surface wave breaking. The results of three-dimensional simulation of wave run-up and breaking under its propagating over a sloping shore are presented in Lubin et all, 2006. The Navier-Stokes equation is used for the simulation with consideration of two-phase air-water medium.

In the framework of shallow water theory in Borisova, 2007, a simulation method for running up of abrupt waves onto a sloping shore and their distribution on the dry sea bed is proposed. Represented is the three-dimensional model of partial destruction of the dam with a local ellipsoidal barrier and a dry sea bed in the lower reach. The paper Delis \& Kazolea, 2008, is devoted to simulation of storm waves and tsunamis propagation within the framework of numerical solutions of nonlinear shallow water equation. The three-dimensional model of stepwise tsunami run-up onto offshore strips is presented in the paper.

The papers Ting \& Kirby, 1996, and Kimmoun \& Branger, 2007 can be noted for results of the experimental run-up investigation. The experimental study of deformation of the breaking surface waves profile in the surf zone is given in Ting \& Kirby, 1996. The stepwise snapshots of the wave profile deformation while propagating on the sloping sea bed before and after the breaking are presented. The results of experimental investigations of the surface wave run-up and breaking process on a sloping shore are analyzed in Kimmoun \& Branger, 2007.

This work is devoted to the three-dimensional simulation of the surface gravity waves run-up in shallow bays. The mathematical model is based on the Navier-Stokes equations. The Azov Sea hydro-physical conditions are used as a shallow water area model. The problems of analytical and numerical modeling of the propagation of nonlinear surface gravity waves for the given shallow-water tank were described in Abbasov, 2010, Abbasov, 2012_1.

The two-dimensional run-up simulation on the basis of the Navier-Stokes equations was carried out in Abbasov, 2013. The three-dimensional models of wave processes are not only the most informative ones, but also allow taking into account the real three-dimensional relief of seabed and shallow bay shore. 


\section{Statement of the Problem}

According to geometry, axis $O x$ the Cartesian coordinate system is superposed with the surface of undisturbed fluid surface and directed to the shore, axis $O y$ is directed along the shore, and axis $O z$ is directed vertically up (Fig. 1). At initial time, the liquid is at rest. The pressure disturbance is set as an impulse at some distance from the shore in $x=0$ point, it is changing in the course of moving off from central point of coordinates according to harmonic law. Let us consider the further water mass motion to the shore.

In order to describe three-dimensional gravity waves on the surface taking into account its viscosity, let us apply Navier-Stokes equations, discontinuity equation for incompressible fluid and hydrodynamic pressure equation (Fletcher, 1988):

(i) Navier-Stokes equations:

$$
\begin{gathered}
\frac{\partial u}{\partial t}+u \frac{\partial u}{\partial x}+v \frac{\partial u}{\partial y}+w \frac{\partial u}{\partial z}=-\frac{1}{\rho} \frac{\partial P}{\partial x}+\mu \frac{\partial^{2} u}{\partial x^{2}}+\mu \frac{\partial^{2} u}{\partial y^{2}}+\eta \frac{\partial^{2} u}{\partial z^{2}} \\
\frac{\partial v}{\partial t}+u \frac{\partial v}{\partial x}+v \frac{\partial v}{\partial y}+w \frac{\partial v}{\partial z}=-\frac{1}{\rho} \frac{\partial P}{\partial y}+\mu \frac{\partial^{2} v}{\partial x^{2}}+\mu \frac{\partial^{2} v}{\partial y^{2}}+\eta \frac{\partial^{2} v}{\partial z^{2}} \\
\frac{\partial w}{\partial t}+u \frac{\partial w}{\partial x}+v \frac{\partial w}{\partial y}+w \frac{\partial w}{\partial z}=-\frac{1}{\rho} \frac{\partial P}{\partial z}+\mu \frac{\partial^{2} w}{\partial x^{2}}+\mu \frac{\partial^{2} w}{\partial y^{2}}+\eta \frac{\partial^{2} w}{\partial z^{2}}+g,
\end{gathered}
$$

(ii) continuity equation for incompressible fluid:

$$
\frac{\partial u}{\partial x}+\frac{\partial v}{\partial y}+\frac{\partial w}{\partial z}=0
$$

(iii) hydrodynamic pressure equation adjusted for depth:

$$
P(x, y, z, t)=p(x, y, z, t)+\rho g z,
$$

where $u, v,-$ horizontal and $w$ - vertical components of water particle motion velocity vector $\mathbf{V}, \rho$ - fluid density, $g$ - gravitation constant, $\mu, \eta$ - horizontal and vertical components of turbulent viscosity coefficient.

Let us consider boundary our problem conditions. There are following conditions on the free surface of fluid:

$$
P(x, y, z, t)=P_{a t m} ; \quad w(x, y, z, t)=\frac{1}{g \rho} \frac{\partial P}{\partial t},
$$

in addition, the vertical velocity is expressed through the pressure derivative by differentiation of expression (5) for hydrodynamic pressure; 
- at the bottom of area conditions impermeable and sliding are assumed:

$$
\begin{gathered}
\frac{\partial}{\partial \mathbf{n}} \mathbf{V}(x, y, z, t)=0, \quad \rho \eta \frac{\partial}{\partial z} u(x, y, z, t)=-\tau_{x}(t) \\
\rho \eta \frac{\partial}{\partial z} v(x, y, z, t)=-\tau_{y}(t), \quad \rho \mu \frac{\partial}{\partial x} w(x, y, z, t)=-\tau_{z}(t)
\end{gathered}
$$

where $\mathbf{n}$ - normal vector to the bottom surface, $\tau_{x}(t), \tau_{y}(t), \tau_{z}(t)$ are the tangential stress components at the fluid bottom;

- the left and right lateral borders boundaries have conditions similar to those on the bottom and backside boundary where there is the impulse source:

$$
\begin{gathered}
\frac{\partial}{\partial \mathbf{n}} P(x, y, z, t)=\alpha, \quad u(x, y, z, t)=u^{(0)} \\
v(x, y, z, t)=v^{(0)}, \quad w(x, y, z, t)=w^{(0)} .
\end{gathered}
$$

Under boundary conditions, the tangential stress components arising due to bottom frictions are calculated per equations (Fletcher, 1988):

$$
\tau_{x}(t)=\rho C_{p}(|\mathbf{V}|) u|\mathbf{V}|, \quad \tau_{y}(t)=\rho C_{p}(|\mathbf{V}|) v|\mathbf{V}|, \quad \tau_{z}(t)=\rho C_{p}(|\mathbf{V}|) w|\mathbf{V}|,
$$

where $C_{p}(|\mathbf{V}|)=\left\{\begin{array}{ll}0,0088 ; & |\mathbf{V}|<6,6 \mathrm{~m} / \mathrm{s}, \\ 0,0026 ; & |\mathbf{V}| \geq 6,6 \mathrm{~m} / \mathrm{s}\end{array} \quad\right.$ - is nondimensional coefficient; parameter $\alpha$ is set based on initial conditions; horizontal and vertical components of the turbulent viscosity coefficient accept following values $\left(0<\mu<1 \mathrm{~m}^{2} / \mathrm{s}\right.$, $\left.0<\eta<1 \mathrm{~m}^{2} / \mathrm{s}\right)$.

At initial time $t=0$ the liquid is at rest, and the following conditions are satisfied: $P(x, y, z, 0)=\rho g z, u(x, y, z, 0)=0, v(x, y, z, 0)=0, w(x, y, z, 0)=0$.

\section{Equation Split}

Approximation of initial equations per time variable is performed by method of splitting according to physical processes (Harlow \& Welch, 1965). Using such method, three-stage calculation is carried out:

(i) at the first stage, field of velocities is calculated based on equations;

$$
\begin{gathered}
\frac{u^{n+\sigma}-u^{n}}{\tau}+u \frac{\partial u}{\partial x}+v \frac{\partial u}{\partial y}+w \frac{\partial u}{\partial z}=\mu \frac{\partial^{2} u}{\partial x^{2}}+\mu \frac{\partial^{2} u}{\partial y^{2}}+\eta \frac{\partial^{2} u}{\partial z^{2}} \\
\frac{v^{n+\sigma}-v^{n}}{\tau}+u \frac{\partial v}{\partial x}+v \frac{\partial v}{\partial y}+w \frac{\partial v}{\partial z}=\mu \frac{\partial^{2} v}{\partial x^{2}}+\mu \frac{\partial^{2} v}{\partial y^{2}}+\eta \frac{\partial^{2} v}{\partial z^{2}}
\end{gathered}
$$




$$
\frac{w^{n+\sigma}-w^{n}}{\tau}+u \frac{\partial w}{\partial x}+v \frac{\partial w}{\partial y}+w \frac{\partial w}{\partial z}=\mu \frac{\partial^{2} w}{\partial x^{2}}+\mu \frac{\partial^{2} w}{\partial y^{2}}+\eta \frac{\partial^{2} w}{\partial z^{2}}+g,
$$

(ii) at the second stage, pressure is calculated;

$$
\frac{\partial^{2} P}{\partial x^{2}}+\frac{\partial^{2} P}{\partial y^{2}}+\frac{\partial^{2} P}{\partial z^{2}}=\frac{\rho}{\tau}\left(\frac{\partial u^{n+\sigma}}{\partial x}+\frac{\partial v^{n+\sigma}}{\partial y}+\frac{\partial w^{n+\sigma}}{\partial z}\right)
$$

(iii) at the third stage, the field of velocities are specified per pressure:

$$
\begin{aligned}
& \frac{u^{n+1}-u^{n+\sigma}}{\tau}=-\frac{1}{\rho} \frac{\partial P}{\partial x}, \\
& \frac{v^{n+1}-v^{n+\sigma}}{\tau}=-\frac{1}{\rho} \frac{\partial P}{\partial y}, \quad \frac{w^{n+1}-w^{n+\sigma}}{\tau}=-\frac{1}{\rho} \frac{\partial P}{\partial z},
\end{aligned}
$$

where $u^{n}, v^{n}, w^{n}$ - velocity vector components on the current time step;

$u^{n+\sigma}, \quad v^{n+\sigma}, \quad w^{n+\sigma}-$ velocity vector components on the auxiliary time step;

$u^{n+1}, \quad v^{n+1}, \quad w^{n+1}-$ velocity vector components on the next time step.

\section{Construction and Investigation of the Discrete Model}

The discrete finite-volume model with consideration of cell filling coefficient was developed (Abbasov, 2012_2). The computational domain of along spatial directions is a cube. For numerical realization of discrete mathematical model, the uniform mesh is introduced (Holt, 1977), (Samarskiy, 1987):

$$
\begin{array}{r}
\varpi_{h}=\left\{x_{i}=i h_{x}, y_{j}=j h_{y}, z_{k}=k h_{z} ; i=\overline{0 . . N_{x}}, j=\overline{0 . . N_{z}} ; k=\overline{0 . . N_{k}} ;\right. \\
\left.N_{x} h_{x}=l_{x}, N_{y} h_{y}=l_{y}, N_{z} h_{z}=l_{z}\right\},
\end{array}
$$

where $i, k$ - indices in directions $x, y, z ; h_{x}, h_{y}, h_{z^{-}}$spatial steps; $N_{x}, N_{y}, N_{z^{-}}$ quantity of spatial points; $l_{x}, l_{y}, l_{z}-$ spatial dimensions of the area. For approximation of original differential equations along the spatial coordinates, the method of balance is applied.

The equation discrete analogues were obtained to calculate components of velocity vector, pressure field, and also discrete analogues of boundary conditions. The discrete model conservatism was studied. It was found that the law of the integrated pulse conservation for the finite-difference equation mesh analogue was met. 


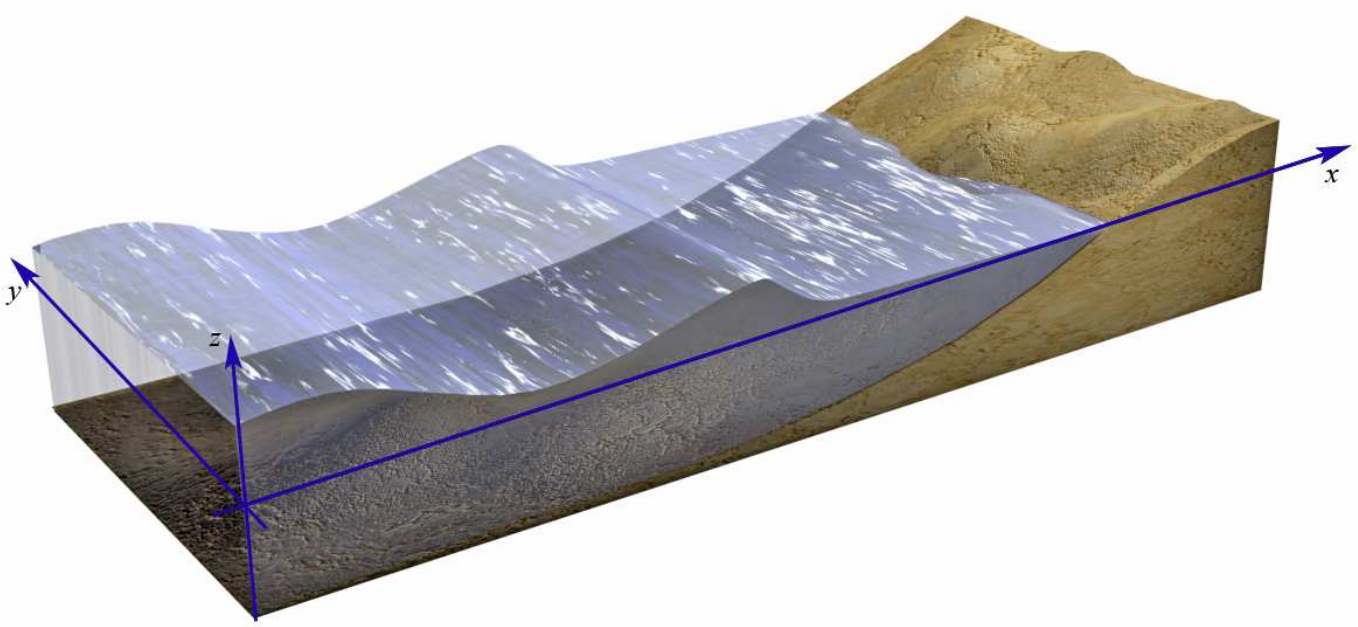

Figure 1: Geometry of three-dimensional problem run-up of the surface gravity waves

Approximation error of finite-difference scheme in the respect of temporal coordinate has the first grade, as to spatial coordinates - second grade. There have been carried out the problem stability study based on the principle of maximum, received time steps and spatial coordinates' boundary conditions.

Discrete equations for calculation of velocity vector components and pressure field are calculated according to implicit scheme. They are calculated according to explicit scheme to specify the velocity field components. The derived discrete equations are resolved by over-relaxation method. The program "3DBayWaves" was developed to calculate the three-dimensional field of velocities and water media pressure field for numerical modeling of run-up and breaking of nonlinear surface gravity wave (Abbasov et all, 2012).

\section{Results of Numerical Modeling and their Analysis}

Let us consider the process of three-dimensional numerical modeling of nonlinear surface gravity waves run-up upon the sloping shores of shallow water tanks. According to statement of problem, the layer of viscous incompressible fluid fills up the certain level of three-dimensional coastal tank, the bottom line transformed to the coastal line (Figure 1).

Before "3DbayWaves" starts-up, the dimensions of the computational mesh, 


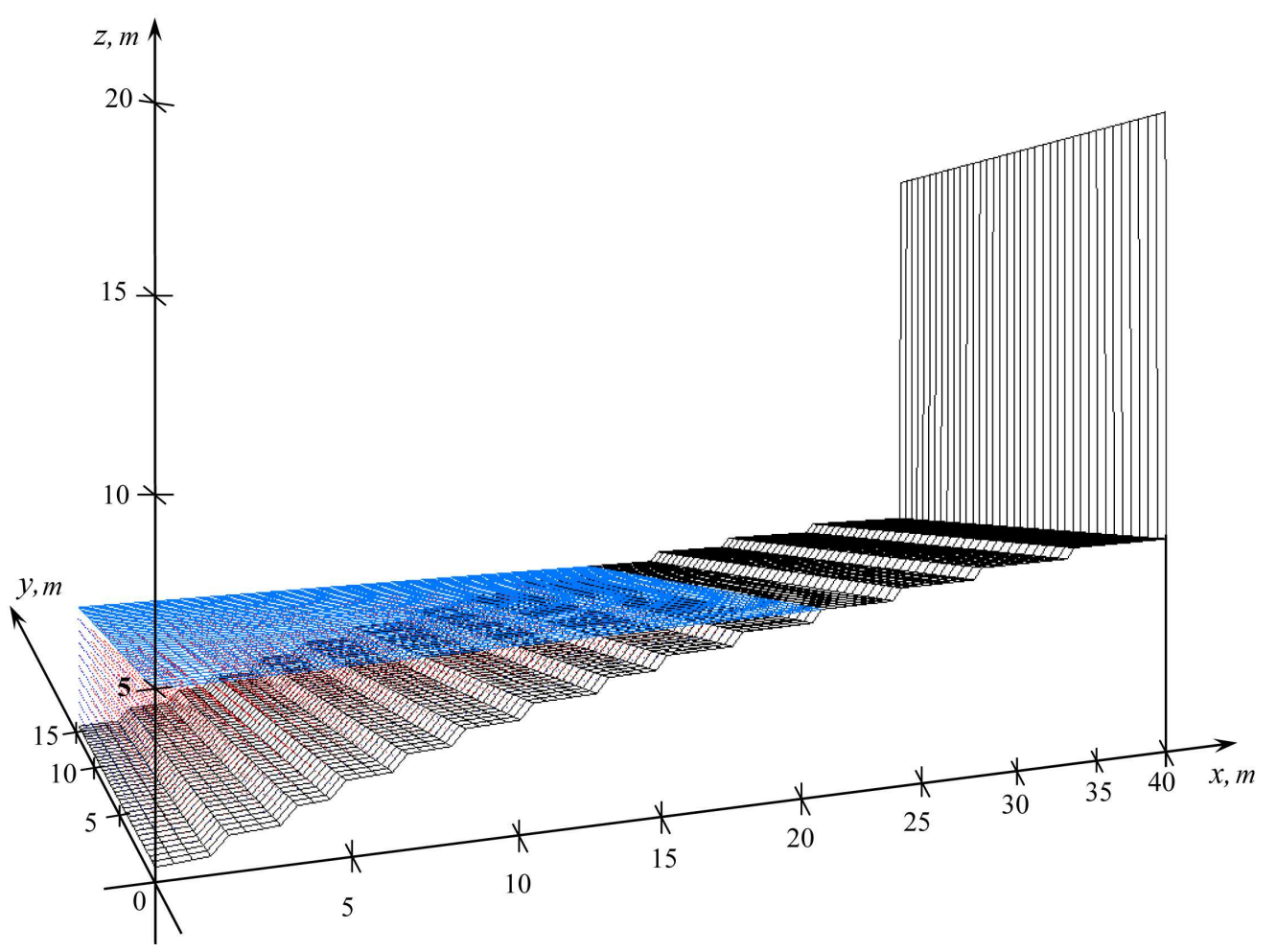

Figure 2: Three-dimensional mesh of shallow tank in the steady state

( $N_{x}, N_{y}, N_{z}$ - spatial boundaries), the three-dimensional geometry of the sloping shore and number of time iterations ( $N_{t^{-}}$upper time boundary) are set. Then the parameters of the surface gravity wave are entered: amplitude $-a$, wave length $-\lambda$, initial depth of fluid at rest $-H$. After setting those data, the three-dimensional mesh for rendering of the tank filled with water is started, which is shown on Figure 2.

The tank bottom is gradually raising and transfers into sloping shore. Bathymetric condition of three-dimensional tank are related to conditions of Taganrog bay in the Azov Sea (Mamykina \& Khrustalev, 1980), (Hydrometeorology, 1991). The three-dimensional tank design was developed with consideration of Azov Sea shores' peculiarities. It refers to coast protection facilities of sloping type with vertical wall.

To provide correctness of physical process, one should keep gradual accumulation of nonlinear effects during surface wave propagation. The mesh size are 1004060 cells, the level of water surface in vertical directions is within 5-25 


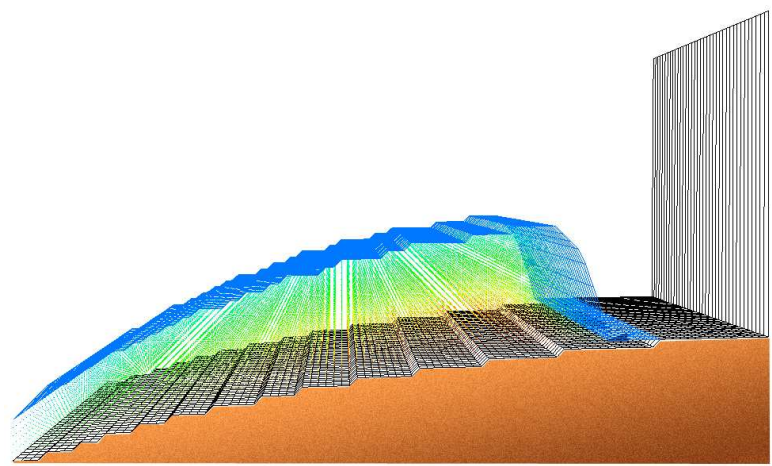

(a)

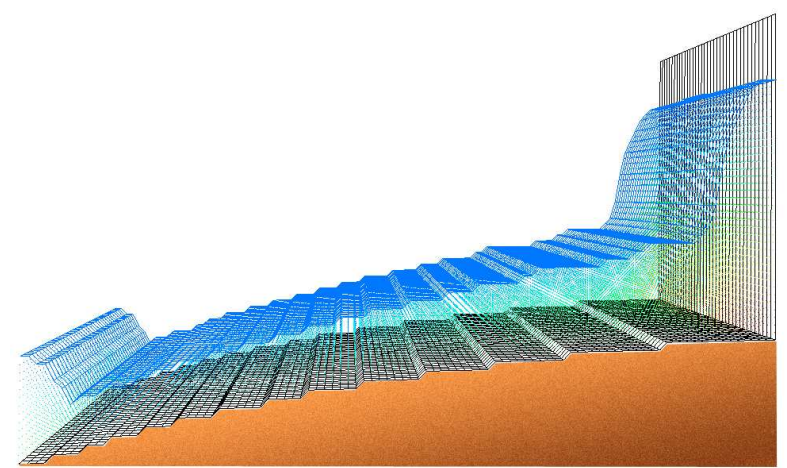

(b))

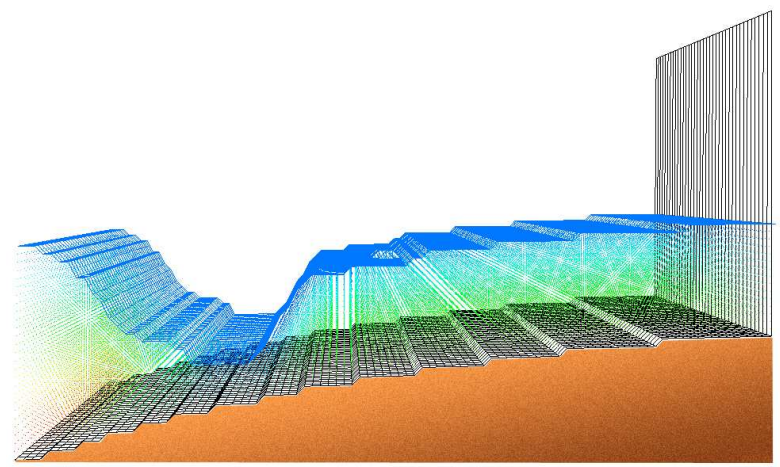

(c)

Figure 3: Sequential stages of surface gravity wave running up at various moments of time $t$ : a) $t=3.7 \mathrm{~s}$; b) $t=6.3 \mathrm{~s}$; c) $t=9.9 \mathrm{~s}$ 
cells. Based on the tank depth of $\mathrm{H} \leq 5 \mathrm{~m}$, the water tank will have the following geometrical dimensions (Figure 2): length $-40 \mathrm{~m}$, width $-15 \mathrm{~m}$, vertical extent - $25 \mathrm{~m}$ (from the bottom), horizontal step length is $h_{x}=h_{y}=0.42 \mathrm{~m}$, vertical one $-h_{z}=0.42 \mathrm{~m}$.

Figure 3 represents the successive process of nonlinear surface gravity wave run-up onto the smooth slope. The media harmonic disturbance with frequency of $f=0.13 \mathrm{~Hz}$ was given on tank backside. Calculations were conducted with the following wave parameters: velocity $c=6,6 \mathrm{~m} / \mathrm{s}$; wave length $\lambda=50 \mathrm{~m}$; amplitude of vertical displacement of water free surface $a=4.2 \mathrm{~m}$; shallow water parameter $k H=0.6$; nonlinearity parameter $\varepsilon=0.8$. The surface gravity wave meets the initial requirement of shallow-water $H / \lambda<1 / 5$ (Whitham, 1974).

With depth decreasing of the three-dimensional tank, the wave reaches the dry coast. Due to growing of nonlinear effects, there is steeping of the wave crest front (Figure 3a). Further the waves front becomes upright and then it breaks-up. While breaking, the wave scatters and hits the vertical wall of the tank, that process is shown on Figure 3b.

Then the water mass starts flowing backwards along the sloping shore, back draft of wave happens Figure 3c. The drifted back wave hits the next wave crest making it even more steeping and accelerating its break-up. In addition, by the time of the next crest upcoming, the level of the tank fluid at rest goes up. The described phases of the surface gravity wave run-up are also valid for surface waves with other initial parameters of shallowness.

For the purpose of checking adequacy of the developed model, the obtained results were compared with existing numerical Lubin et all, 2006 and experimental Kimmoun \& Branger, 2007, data. The comparison showed good concurrence of the obtained results with the existing data in respect of the surface wave main phases of propagation and breaking up.

\section{References}

[1] Y. Watanabe, H. Saeki. Three dimensional large eddy simulation of breaking waves, Coast. Eng. J. 41 (1999), 281-301. doi: $10.1142 / \mathrm{S} 0578563499000176$

[2] P. Lubin, S. Vincent, S. Abadie, J.-P. Caltagirone. Three-dimensional large eddy simulation of air entrainment under plunging breaking waves, Coastal Engineering, 53 (2006), 631-655. doi:10.1016/j.coastaleng.2006.01.001

[3] N. M. Borisova. On modeling of hydraulic bore propagation at incline bank, Siberian Journal of Numerical Mathematics, 10 (2007), 43-60. 
[4] A. I. Delis, M. Kazolea, N. A. Kampanis. A robust high resolution finite volume scheme for the simulation of long waves over complex domains, Int. J. Num. Meth. In Fluids, 56 (2008). 419-452, doi:10.1002/fld.1537

[5] F.C.K. Ting, J.T. Kirby. Dynamics of surf-zone turbulence in a spilling breaker, Coastal Engineering, 27 (1996). 131- 160. doi:10.1016/0378$3839(95) 00037-2$

[6] O. Kimmoun, H. Branger. A PIV investigation on laboratory surf-zone breaking waves over a sloping beach, J. Fluid Mech, 588 (2007). 353-397. doi:10.1017/S0022112007007641

[7] I.B. Abbasov. Modelling of nonlinear wave phenomena on a shallow water surface. Moscow. Fizmatlit (2010).

[8] I.B. Abbasov. Numerical simulation of nonlinear surface gravity waves transformation under shallow-water conditions, Applied Mathematics, 3 (2012). 135-141. doi:10.4236/am.2012.32021

[9] I.B. Abbasov. Mathematical modelling the runup of nonlinear surface gravity waves, International Journal of Pure and Applied Mathematics, $\mathbf{8 2}$ (2013). 132-142.

[10] C.A. Fletcher. Computational techniques for fluid dynamics, V.1, Berlin, Springer-Verlag (1988).

[11] F.H. Harlow, J.E. Welch. Numerical calculation of time-dependent viscous incompressible flow of fluid with free surface, Phys. Fluids, 8 (1965). 21822189. doi:10.1063/1.1761178

[12] I.B. Abbasov. Modeling the runup of nonlinear surface gravity waves on the basis of Navier-Stokes equation, Computational continuum mechanics, 5 (2012). 322-326. doi:10.7242/1999-6691/2012.5.3.38

[13] M. Holt. Numerical methods in fluid dynamics. Springer-Verlag (1977).

[14] .. Samarskyi. Introduction to numerical methods. 2-nd pub. Moscow: Nauka. Gl. red. fiz.-mat. lit. (1987).

[15] I.B. Abbasov, I.S. Semyonov, V.V. Tsarevsky. Program of threedimensional modelling of the runup surface waves on shallow water "3DBayWaves": Copyright certificate 2012617087 about the state registration of the computer program. 22.05.2012 declared; 08.08.2012 published. 
[16] V.. Mamykina, Yu.P. Khrustalev. Coastal Zone of the Azov Sea. Rostovon-Don. (1980).

[17] Hydrometeorology and Hydrochemistry of the seas in the USSR. .V. The Azov Sea. S.-Pb.: Gidrometeoizdat (1991). 75-88.

[18] G. B. Whitham. Linear and nonlinear waves. New York: J. Wiley \& Sons (1974). 
